\title{
The Polarization Field for Pulsed Raman Transitions \\ IV
}

B. W. Shore, R. Sacks and T. Kars

Lawrence Livermore National Laboratory

\begin{abstract}
A bstract
This memo discusses the induced colarization field that occurs in the presence of Raman processes, and the propagation equations that result from this field.

Section IV.I summarizes the relationship between the macroscopic polarization field and the microscopic dipole-moment expectation value. It summarizes expressions for the induced dipole moment that result from the adiabatic elimination of non-resonant molecular transitions, to produce an effective two-photon (Raman) Hamiltonian.

In Section IV.2 we show that the polarization field has a similar mode expansion to the electric field.

Using this result, we describe in Section IV.3 the equations for pulse propagation of the electric field. These equations involve a generalized gain matrix and mode velocity, as well as a refractive index, each of which depends upon position and time.

Section IV.4 summarizes these results and exhibits succinctly the pulse propagation equations in the plane-wave slowly-varying envelope approximation. The equations presented here must be supplemented with excitation equations (or by steady-state results) for the molecules.
\end{abstract}

The material presented here is a portion of a more extensive treatment of propagation to be presented separately.

\section{DISCLAIMER}

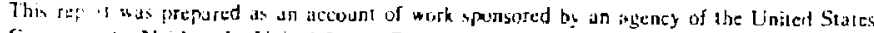

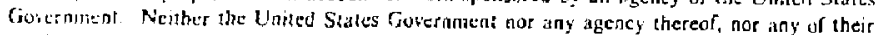
cmplayees. makes all, warranty, cxpress ar implied, or assumes any legal liatality or responsjbility for the accuracy, zompleteness. or uselulness of any information, apparatus, producl, or process distlosed. ar represents that its use would not infringe privately owned rights. Reference hercin to any specilic commercial producl, process, or serwice hy trade name, Itademark, manufacturer, or otherwise does not necessarily constitute or imply its endorsement, recommiendation, or fayoring by the Uniled States Government or any agency thereof. The views and opinions of authors expressed nerein do not necessarily state of reflect those of the United Stites Government or any agency thereof.
}

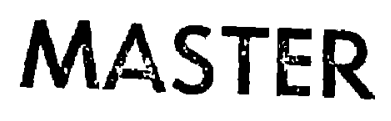




\section{\$JV.I The Polarization Field}

Electromagnetic wave propagation, of which pulsed rays are a particular case, may be described by the wave equation

$$
\left[\nabla^{2}-\frac{1}{c^{2}} \frac{\partial^{3}}{\partial^{2}}\right] E=-\frac{4 \pi}{c^{2}} \frac{\partial^{2}}{\partial t^{2}} P \text {. }
$$

where $E \mp E(r, t)$ is the solenoidal (transverse) part of the electric field and $P \mp P(r, t)$ is the solenoidal polarization field. The polarization field $P$ that acts as a source term for radiation propagation is the macroscopic average dipole-moment distribution; it is the electric dipole moment per unit volume. In the simple case of a uniform distribution of ideatical molecules we write the polarization $\mathbf{P}$ as the density of molecules $\mathcal{N} \equiv \mathcal{V}(r, t)$ times the dipole moment per molecule $\alpha d\rangle$,

$$
\mathbf{P}=\mathcal{N}<\mathbf{d}>
$$

The generalization to multiple species is noted below. This microscopic moment <d is the expectation value of the molecular dipole moment operator $d$ at position $r$. Like any vector, this operator has three components, and can be expressed in a fixed laboratory reference frame in terms of Cartesian unit vectors in that frame,

$$
d=\sum_{i} d_{i} e(i) \text { where } d_{i}=\text { d.e }(i)
$$

More generally, the dipole moment can be expressed in terms of whatever unit vectors one chooses for describing the field.

\section{The Field Mode Expansion}

We are interested in directed pulses of radiation, such as may be described by the following expression for the positive frequency part of the field as a superposition of envelope functions $\mathcal{E}_{\lambda}(r, t)$ times traveiling-wave carriers:

$$
E^{(+)}(r, t)=\sum_{\lambda} E^{(+)}{ }_{\lambda}(r, t)=\frac{2}{2} \sum_{\lambda} c(\lambda) \delta_{\lambda}(r, t) \exp \left[i \mathbf{k}_{\lambda} \cdot r-i \omega_{\lambda} t\right)
$$

Here the label $\lambda$ that jdentifies the mode specifies the unit polarization vector $\theta(\lambda)$, the propagation vector $k_{\lambda}$, and the frequency $\omega_{\lambda}$.

Our purpose in the present memo is to describe a similar mode expansion for the polarization field and to separate the polarization field into a linear and a nonlinear portion. We obtain an explicit expression for the connection between $k_{\lambda}$ and $\omega_{\lambda}$ and a propagation equation for the pulse envelope function. 
The Polarizability

When the dipole moment is induced by a static electric field $E$ one introduces a polarizability tensor a such that

$$
\text { <d }=\text { a.E }
$$

This vector equation abbreviates the set of three equations

$$
\left\langle d_{i}\right\rangle=\sum_{j} a_{i j} E_{j}
$$

for three components of the vectors $\mathbf{d}$ and $\mathbf{E}$. Somewhat more cererally one may considers a time varying (rypically monochromatic) electric field $\mathbf{E}(t)$ and introduces a time or frequency-dependent polarizability tensor. We shall examine the definition of such a tensor below.

The static polarizability expresses the linear response of molecule to an external static uniform electric field. In the presence of such a field the molecule acquires an additional increment of energy $\hbar \delta$,

$$
\hbar E W=-\langle d\rangle . E=-E \cdot a \cdot E
$$

and so a measurement or a calculation of this energy shift provides a value for the static polarizability. This energy increment depends upon the nanure of the moleculas states that are involved in the expectation value of the dipole moment, and a more complete notation would indicate this dependence.

We shall see below the generalization of the polarizability tensor to treat time varying fields, and pulse mode expansions of the electric field.

\section{The Dipole Moment Distribation}

When there is only one type of molecule, and each molecule is described by a pure quantum state $|\Psi\rangle$, then the dipole-moment expectation value is

$$
\langle d\rangle=\left\langle\mathbb{U} \mathbf{d} \Psi_{2}\right.
$$

Unless the molecule is prepared in, and remains in, an eigenstate of the energy operator, this expectation value varies with time: one has $\langle d\rangle=\langle d(1)\rangle$. Such variations certainly occur in the situations of interest, viz the excitation of molecules by pulses of radiation. The time variation may be placed into the state vector (thereby defining the Schroddinger picture)

$$
\langle d(t)\rangle=\langle\Psi(t) \| d \mid \Psi(t)\rangle
$$

or in the dipole operator (thus establishing thie Heisenberg picture)

$$
\langle\mathrm{d}(t)\rangle=\langle\Psi \cdot \mathrm{d}(t) \mid \Psi\rangle
$$

or in a combination of the two (as in the Dirac picture). 
More generally, the medium consists of a variety of types of molecules, say different chemical species, different initial states of excitation, disfered velocities, etc. Let the number density of type $a$ be

$$
r_{a}=p(a) \mu_{0}, \quad \sum_{a} p(a)=1
$$

For matter in local inermodynamic equilibrium the probabilities $p(a)$ are given by appropriate Boltzmann factors and partition functions. More generally, these probabilities are solutions to appropriate molecular rate equations (in the absence of the pulsed radiation). In any event, the dipole expectation value then takes the form

$$
\left.\langle d\rangle=\sum_{\alpha} p(\alpha)\langle d\rangle_{\alpha}=\sum_{\alpha} p(\alpha)<\Psi_{\alpha}|d| \Psi_{\alpha}\right\rangle
$$

The properties of matter exposed to radiation will depend upon time, as a result of radiation-induced excitation. The properties will also generally depend upon spatial location. In part this variation occurs because of initial inomogeneities in the oistribution of matter, and in part it occurs because the radiation freld changes with position, and so the Hamiltonian governing the time evolution of the state vector will change with position. We can allow for such variations by introducing, as a parameter, the location $r$ of the molecular center of mass, and writing the dipole expectation value in the form

$$
\langle d(r, t)\rangle=\sum_{\alpha} p(\alpha, r, t)<\Psi_{\alpha}(r, t), d\left|\Psi_{\alpha}(r, t)\right\rangle
$$

The polarization field may be written as

$$
P(r, t)=\sum_{\alpha} \mathcal{N}_{\alpha}(r, t)<\Psi_{\alpha}(r, t) d \mid \Psi_{\alpha}(r, t)
$$

The dependence of the polarization field upon space and time occurs for two reatuns. Fist, the factor $\mathcal{N}_{\alpha}(r, t)$, the density of molecules of type $a$, changes with time and position as a result of initial concentration gradients and subsequent macroscopic motions of molecules. These variations of density occur on time and distance scales that are very large compared with those that occur in treating propagation. They are described by appropriate macroscopic hydrodynamic equations. Second, the state vector $\Psi(r, t)$ changes with time as a consequence of radiative excitation. It will change with position because the excitation Hamiltonian changes with position. It is the changes in this state vector that we determine by means of the coupled MaxwellSchrodinger equations. 


\section{Basis Expansion}

To evaluate such ac expression one requires some means of characterizing the molecular state vector. This is always done by expressing the molecular sate vector, or wavefunction, in terms of some tractable set of basis states. In general, we introduce a set of molecular basis states $\phi_{m}$ and write the statevector at time $t$ for: molecule whose center of mass is at $r$, as

$$
\Psi(r, t)=\sum_{m} \phi_{m} C_{m}(r, t) \exp \left[-i \zeta_{m}(r, t)\right]
$$

Ideally the suramation goes aver a complete sel of molecular states. In practice we include only some finite approximation to such a set. The exponential phases $\zeta_{m}(r, t)$ are chosen a priori with the intention of incorporating at least part of the identifiable dependence on position and time, so that the probability amplitudes $C_{m}(r, t)$ are more slowly varying.

\section{The Phases}

Although in principle the phases may be chosen as arbitrary functions of space and time, in practice one assumes separability and writes

$$
\zeta_{m}(r, t)=w_{m} t-K_{m} \cdot r .
$$

The frequency $\omega_{m}$ may be taken as zero (thereby defining the Schrodinger picture) or as the molecular frequency $W_{m}$ corresponding to the unperturbed energy eigenvalue $h W_{m}$ of state $\phi_{m}$ (thereby defining the Dirac picture), or it may be taken as some combination of radiation-field frequencies (therby defining a rotating wave picture). The latter choice allows us to remove from the Hamiltonian the time variations associated with near-resonant carrier frequencies, leaving an effective Hamillonian that varies in time only because of slower changes in pulse envelopes. For descriptions of radiatinn-induced motions (such as cooling or trapping of molecules by radiation), it is often useful to incorporate into the molecular energies the center-ofmass kinetic energy $\mathrm{Mv}^{2} / 2$ associated with molecular center-of-moss velocity $\mathbf{v}$. When excitation is largely attributable to photon absorption (rather than collisions with massive particles), one may wish to define phases that include appropriately modified kinetic energies corresponding to internal excitation states.

The wavevector $\mathbf{K}_{\mathbf{m}}$ allows us to remove a portion of the sparial variation of the Hamiltonian that originates in plane-wave carriers. It amounts to the introduction of a state-dependent center-of-mass momentum for the molecule. For molecules initially at rest in the ground state $\phi_{0}$ we would take $\mathbf{K}_{0}=0$, whereas for a moving molecule $\hbar K_{0}$ may be taken as the molecular momentum Mv. The remaining excited-state values represent momentum transferred to the molecule as a consequence of photon absorption from the field. In a photon-number representation of the field differences of these vectors represent molecular momentum changes as a resuh of photon absorption and emission ( $c$ f. the discussion in Bernhardt and Shore, 1981). In the classical description of the fields used here, differences of wavevectors cocur when ve evaluate products of electric field mode amplitudes. We shall be interested in propagation along a beam. Under those circumstances the most convenient choice for $\mathbf{K}_{t}$ will be a vector along the primary propagation axis, say the $z$ direction, so that the phase 
reads

$$
\zeta_{m}(t, t)=w_{m} t-K_{m} 2
$$

The use of such phases is equivalent to introducing a delay time

$$
T_{\mathrm{m}}=\frac{K_{\mathrm{m}}}{w_{\mathrm{m}}}=\frac{2}{v_{\mathrm{m}}}
$$

and writing the state-vector expansion coefficient as

$$
C_{m}(r, t) \exp \left[i\left(K_{m} z-\omega_{m} t\right)\right]=C_{1 j}(r, t) \exp \left\{-i \omega_{m}\left(t-T_{m}\right)\right]
$$

When all of wavevectors $K_{m}$ and the frequencies $\omega_{m}$ have a common ratio (the phase velocity $v_{m}$ and the delay time $T_{m}$ are independent of $m$ ), then the phase choice is equivalent to introducing a retarded time $r=t-T_{m}$ as the temporal argument of the probability amplitude: we take the local zero-point of time (at a given molecule) to ccincide with the arrival of a fiducial mark moving with the phase velocity $v=$ $\omega_{m} / K_{m}$. The expansion coefficient then becomes

$$
C_{m}(z, 1) \exp \left[i\left(K_{m} z-w_{m} t\right)\right]=C_{m}(r, \tau+T) \exp \left[-i \omega_{m} r\right] .
$$

where $r=t-z / v$. Although it is possible to use a different time scale for each probability amplitude (chereby introducing a different time window for each pulse), such a procedure complicates the equations of mojon.

It should be stressed that the phases $\zeta_{\mathrm{m}}(r, t)$ are introduced solely to simplify subsequent equations; ideally they produce amplitude functions that vary more slowly with space and time. Any injudicious choice of explicit phase will ultimately be compensated by a phase in the amplitude $C_{m}(r, 1)$.

\section{$P$ and $Q$ states}

For application to Raman processes we can divide the molecular basis states into two sets. In the first set we place the ground state and those low-lying rotational (or rotational and vibrational) states that connect with the ground state by various Raman processes. (These are processes in which one photon is absorbed and a different photon is emitted.) Let us call this set the $P$ set. They are the states $\phi_{p}$. The remaining states form the $Q$ set, and are denoted $\phi_{4}$. They are each orthogonal to each of the $P$ states. The Dirac-picture expression for the molecular state vector $\Psi(x, t)$ is

$$
\Psi(r, t)=\sum_{p} C_{p}(r, t) \exp \left(i K_{p} \cdot r-i W_{p} t\right) \phi_{p}+\sum_{q} C_{q}(r, t) \exp \left(i K_{q} \cdot r-i W_{q} t\right) \phi_{q}
$$

The constant vectors $K_{p}$ are introduced so that we may remove a porion of the posjtion dependence of the state vector. He take them to be directed along the dominant propagation axis, but to be othernise arbitrary for the present.

\section{The Dipole Moment Expansion}


We have assumed that the $Q$ states are non resonant, and as a consequence they participate only indirectly in the dynamics. Therefore we can neglect contributions to the dipole-moment expectation value that come frow transitions between $Q$ states, and write the dipole monent expectation value as

$$
\begin{aligned}
\langle d(r, t)\rangle & =2 \text { Re } \sum_{p p^{\prime}}\left\langle p^{\prime}|d| p\right\rangle C_{p^{\prime}}(r, t)^{*} C_{p}(r, t) \exp \left[i\left(K_{p}-K_{p^{\prime}}\right) \cdot r-r\left(W_{p^{\prime}}-W_{p^{\prime}}\right) t\right] \\
& +2 \text { Re } \sum_{p q}\left\langle p^{\prime}|d| q\right\rangle C_{p}(t)^{*} C_{q}(t) \exp \left[i\left(K_{q}-K_{p}\right) \cdot r-i\left(W_{q}-W_{p}\right) t\right]
\end{aligned}
$$

The phase factors that appear here are just those that were introduced in the basisstate expansion of $\Psi(r, t)$, and which defined the Dirac picture for the expansion coefficients. Let us, for the moment, assume that there are no electric-dipole moments between the basis states of the $P$ set, so that all direct, single-photon El transitions link the $P$ states with the $Q$ states. If the time variations of the amplitudes are not 100 rapid, we can separate this expectation value into positive and negative frequency parts according to which of the two positive numbers $W_{p}$ and $W_{Q}$ is larger. We have, in this simpie case, the expression

$$
\left\langle\mathrm{d}^{(+)}(r, t)=\sum_{p<q}\langle p|d| q\rangle C_{p}(r, t)^{*} C_{q}(r, t) \exp \left[i\left(K_{q}-K_{p}\right) \cdot r-i\left(W_{q}-W_{p}\right) t\right]\right.
$$

That is, the dipole moment appears as a succession of carrier travelling waves, modulated spatially and temporally by bilinear products of probability amplitudes. By hypothesis the vectors $K_{p}$ are directed along the primary propagation axis, and so that is the direction of these plane waves. In turn, it follows that the polarization field has positive frequency components, at fixed position $r$,

$$
P^{(+)}(r, t)=\mathscr{N} \sum_{p<q} \operatorname{spdd\varphi } C_{p}(r, t) c_{q}(r, t) \exp \left[i\left(K_{q}-K_{p}\right) s-i\left(W_{q}-W_{p}\right) t\right]
$$

These two expressions assume that the frequencies $W_{p}$ and $W_{q}$ dominate the time dependence. We shall consider below situations when this is not the case.

This expression for the polarization field must be cast into the form of a mode expansion in order to identify the portion that acts as a source for a mode of the electric field. That identification, taken with the Schrodinger equation (or some equivalent molecular equation), provides the traditional basic set of equations governing pulse propagation. They are often applied to propagation of pulses whose carrier frequency is near resonance with one (or more) of the molecular frequency differences $W_{q}-W_{p}$. Under those circumstances, of near-resonant single-photon excitation, the amplitudes $C_{p}(r, t)$ and $C_{q}(r, t)$ bave similar time variations, and these are 
much slower than the frequency $w_{Q}-w_{p} \pm w_{\lambda}$. However, when the pulse carrier frequedcies are far from resonance with theic single-photon trancitions, thea it is no longer possible to regard $C_{q}(r, 1)$ as slowly varying in lime. This is the situation of interest to us bere. We must find an alternative expression for the dipole moment, and the polarization field, that provides a simple separation of a slowly varying envelope and a carrier frequency.

\section{The Adiabatic Approximation}

Our basic assumption is that all Q-states are far from resonance. We are then able, by adiabatic elimination, to express each coefficient $C_{C}(r, t)$ at time $t$ in terms of all of the P-state coefficients at this same time. We have described this procedure in a previous section [cf. section excit]. There we showed that the connection matrix $M^{\lambda}{ }_{q p}(t)$ in the expression

$$
c_{q}(r, t)=\sum_{\lambda p} M^{\lambda}(r, t) C_{p}(r, t)
$$

has elements

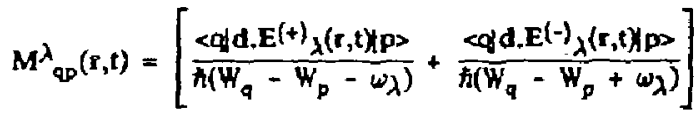

$$
\begin{aligned}
& x \exp \left[i\left(K_{p}-K_{q}\right) \cdot r-i\left(W_{p}-W_{q}\right) t\right] .
\end{aligned}
$$

As discussed elsewhere (see section excit) this expression for the matrix $M^{\lambda}(r, t)$, and in turn the expression for $C_{q}(r, t)$, rests upon the following approximations. First, we conside: only dirzet electric-dipole transitions (the essential states approximation). Second, we introduce a field mode expansion in which, for each mode, the Fourier transform is appreciable only within a bandwidth that is much smaller than anj of the energy separations $W_{q}-W_{p}$ (the non-resonant approximation). Third, we assume that the Fourier inansform of the coefficient $C_{p}$ is appreciable only within a bandwidth that is much swaller than any frenuency $W_{q}-W_{p}-\omega_{\lambda}$.

We can use the preceding adiabatic approximation for the $Q$ states to express the dipole moment as

$$
\begin{aligned}
& \langle d(r, t)\rangle=2 R_{e} \sum_{p p^{\prime} q \lambda} C_{p^{\prime}}(r, t)^{*}\left\langle p^{\prime}|d| q\right\rangle M_{q p}^{\lambda}(r, t) C_{p}(r, t) \\
& x \exp \left[i\left(K_{q}-K_{p^{*}}\right) \cdot r-i\left(W_{q}-W_{p^{*}}\right) t\right]
\end{aligned}
$$

The time dependence of the matrix $\left.M \mathcal{X}_{r, t}\right)$ is, in principle, readily calculable, given the time dependence of the field-mode amplitude $\mathscr{E}_{\lambda}(r, t)$. Thus the dipole moment can be determined once we have obtained the P-space expansion coefticients $C_{p}(r, t)$. 


\section{The Densiiy Matrix}

The dependence of the dipole moment upon molecular excintion can be extracted by means of a molecular statistical matrix or density matrix. In the Dirac picture, and within the set of $P$ states, the elements of this matrix $\rho$ are

$$
\rho_{p p^{\prime}}(r, t)=C_{p}(r, t) C_{p^{\prime}}(r, t)^{*}=\left[\rho_{p^{\prime} p}(r, t)\right]^{*}
$$

Using this matrix we write the dipole-moment expectation value as

$$
\left\langle d(r, t)=2 \operatorname{Re} \sum_{p p^{\prime} q \lambda} P_{p p^{\prime}}(r, t)<p\right\} d \mid q>M_{q p^{\lambda}}^{\lambda}(r, t) \exp \left[i\left(K_{q}-K_{p^{\prime}}\right) \cdot r-r\left(W_{q}-W_{p^{\prime}}\right) t\right]
$$

The time dependence of the dipole moment occurs in three places first, in the exponential that appears expliciuly here; second, from similar exponentials in the matrix $M^{\lambda}(r, t)$, and third, from other implicit exponentials in the density matrix $P_{\mathrm{PF}}(r, t)$. All of these must be cossidered together, in order to extract the explicit time dependence of the dipole moment.

\section{The Polarizibility Opentor}

The expression for the dipole moment that obtains upon substituting expression (IV.1-25) into Eqns. (TV.1-26) or (IV.1-28) can be simplified by introducing the the polarizability operator $X(\omega)$, whose Cartesian components $X_{\mathrm{ij}}(\omega)$ are defined by the molecular matrix elements

$$
\left\langle p^{\prime}\left|x_{i j}(\omega)\right| p\right\rangle=\sum_{q}\left[\frac{\left\langle p^{\prime}\left|d_{j}\right| q><q\left|d_{j}\right| p\right\rangle}{E_{q}-E_{p}-\hbar \omega}+\frac{\left.\Phi^{\prime}\left|d_{j}\right| \varphi<q\left|d_{i}\right| p\right\rangle}{E_{q}-E_{p^{\prime}}+\hbar \omega}\right]
$$

It is possible to exhibit an explicit expression for the operator $\mathbf{X}_{i j}(\omega)$ by converting the denominators into time integrals. The energies appearing in the exponentials may be replaced by energy operators (the field-free molecular Hamitionian). The result may be written

$$
X_{i j}(\omega)=\operatorname{Lim}_{\leftrightarrow 0} \frac{i}{\hbar} \int_{0}^{\infty} \mathrm{dt} \exp (-i \omega t-t)\left[\dot{d}_{i}, d_{j}(t)\right]
$$

The Hermitian operator $d_{j}(t)$ that appears here is a Cartesian component of a Diracpicture dipole-moment operator, obtainable by action of the free-rolecule Liouville operator $\mathbf{L}^{0}$

$$
d_{j}(t)=\exp \left(\pi H^{0} / h\right) d_{j} \exp \left(-s H^{0} / h\right) \equiv \exp \left(i O^{O}\right) d_{j}
$$

This operator may be regarded as the solution to the Heisenberg equation 


$$
\frac{\partial}{\partial t} d(t)=L^{*} d(t)=\frac{i}{\lambda}\left[H^{0}, d(t)\right]
$$

\section{General Expression for Dipole Moment}

By introducint the polarivibility operator $X(\omega)$ and using the properties

$$
\rho_{p p}(r, t)^{*}=\rho_{p^{\prime} p}(r, t), \quad\left\langle p|d| \phi^{*}=\langle q| \text { 셔 } p^{\prime}\right\rangle .
$$

we can write the expectation value of the induced dipole moment as

$$
\begin{aligned}
\langle d(r, t)\rangle= & \sum_{p \lambda p^{\prime}} \rho_{p p^{\prime}}(r, t) \exp \left[i\left(K_{p^{\prime}}-K_{p^{\prime}}\right) \cdot r-i\left(W_{p^{\prime}}-W_{p^{\prime}}\right) t\right] \\
& x\left[\rho^{\prime}\right] X\left(w_{\lambda} \times p>\cdot E^{(+)}{ }_{\lambda}(t)+\langle p| X\left(-w_{\lambda}\right) p>. E^{(-t}{ }_{\lambda}(t)\right]
\end{aligned}
$$

This is the general expression for the induced dipole momenl in the adiabatic (or non-resonant) approximation. Note that because of the complex conjugation properties of $p_{p_{p}}=\left(\rho_{P^{\prime}}\right)^{*}$ and $E^{(-)}=\left(E^{(+)}\right)^{*}$ we can write this expression as twice the real part of the positive frequency portion. If we also include the possibility of singlephoton resonances, via dipole transitions, we obtain the more general formuk

$$
\begin{aligned}
& \left.\langle d(r, t)\rangle=2 \text { Re } \sum_{p^{\prime}<p^{\prime}} P_{p^{\prime}}(r, t)<p^{\prime}|d| p\right\rangle \exp \left[i\left(K_{p^{\prime}}-K_{p^{\prime}}\right) \cdot r-i\left(W_{p^{\prime}}-W_{p^{\prime}}\right) t\right] \\
& +2 \text { The } \sum_{p \lambda p^{\prime}} \rho_{p p^{\prime}}(r, t)<p^{\prime}\left|X\left(\omega_{\lambda}\right)\right| p>. E^{(+)} \lambda_{\lambda}(r, t) \exp \left[i\left(K_{p}-K_{p^{\prime}}\right) \cdot r-i\left(W_{p}-W_{p^{\prime}}\right) t\right]
\end{aligned}
$$

The first term is absent if there are no single-photon dipole transitions amongst the $P$ states. We shall assume this is the case.

\section{Corrections to Adiabatic Elimination}

The procedure of adiabatic elimination used to produce these results requires that the pulse bandwidth be narrow compared with the single-photon resonance frequency between a typical $P$ state and the lowest lying $Q$ state. Corrections to this approximation involve time derivatives of the pulse envelope. In place of the second part of Eqn. (IV.1-35) we obtain the expression

$$
\langle d(r, t)\rangle=R_{a} \sum_{p \lambda p^{\prime}} \rho_{p p^{\prime}} \cdot(r, t) \exp \left[i\left(K_{p}-K_{p^{\prime}}+K_{\lambda}\right) \cdot r-i\left(W_{p}-W_{p^{\prime}+\omega_{\lambda}}\right) t\right]
$$




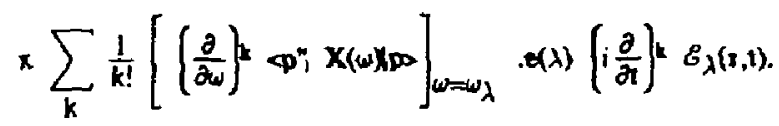

This altered expression for the dipole moment accompanies a corresponding alteration of the Hamiztonian.

\section{The Schrodinger Picture}

$T_{1}$ a density matrix that appears above is in the Dirac picture. That is, the rapidly varying phase factors exp $\left(i K_{p}, r-r W_{p} t\right)$ have been extracted explicitly, to leave a more slowly varying density matrix $\alpha(r, t)$. If we had not extracted these phases we would have had the density matrix $\tilde{p}(x, t)$ in the Schrodin?er picture:

$$
\begin{aligned}
\vec{\rho}_{p^{\prime}}(r, t) & =\rho_{p^{\prime}}(r, t) \exp \left[i\left(K_{p^{\prime}}-K_{p^{\prime}}\right) \cdot r-i\left(W_{p^{\prime}}-W_{p^{\prime}}\right) t\right] \\
& \left.=\left\langle\psi_{p} \mid \Psi(r, t)\right\rangle\langle\Psi(r, t)\rangle \psi_{p^{\prime}}\right\rangle .
\end{aligned}
$$

Using this picture we write the dipole moment as

$$
\left\langle\mathrm{d}(r, t)=2 \text { Ge } \sum_{\bar{\lambda}} F_{\operatorname{mad}} \tilde{\rho}(r, t) X\left(\omega_{\lambda}\right)\right], E^{(+)} \lambda(r, t)
$$

In the low-frequency regime we can approximate $X(\omega)$ by $X(0)$, and then we have the expression

$$
\left.\langle\mathrm{d}(\mathbf{r}, \mathbf{t})\rangle=g_{\mathrm{raod}} \tilde{\boldsymbol{\rho}}(\mathbf{r}, \mathrm{t}) \mathbf{X}(0)\right] . \mathrm{E}(\mathbf{r}, \mathbf{t}) .
$$

It should be emphasized that although this formula appears to present a linear reiationship between dipole moment and electric field (or between polarization field and electric field), the density matrix will depend upon molecular response to the electric field, and so in the general case the rebationship is actually nonlinear.

\section{The Polarizability}

Let us define a frequency-dependent polarizability tensor a $(\omega)$ associated with a monochromatic field $\mathbf{E}_{\omega}$ as

$$
\left.\left\langle d(r, t\rangle=\alpha(\omega) \cdot E^{(+)}{ }_{\omega}(r, t)+\alpha-\omega\right) \cdot E^{(-)}\right)_{\omega}(r, t) .
$$

The present description of pulse-induced dipole moments fits this pattern if we treat steady-state excitation by monochromatic light. The polarizability tensor is then

$$
\left.\alpha(\omega)=\sum_{p p^{\prime}} \tilde{\rho}_{p p^{\prime}}(r, t)\langle p \mid X(\omega) p\rangle=\sigma \operatorname{raod} \tilde{p}(r, t) X(\omega)\right\}
$$

Again we note that the implicit field dependence of the density matrix introduces an implicit dependence of the polarizability tensor upon the electric field. This expres- 
sion for the polarizibility tensor becomes, in the rotating-wave picture,

$$
\left.\alpha(\omega)=\sum_{p p^{\prime}} p_{p p^{\prime}}(r, t)<p^{\prime}\right\} X(\omega), p>\exp \left[i\left(K_{p}-K_{p^{\prime}}\right) . r-i\left(W_{p}-W_{p^{\prime}}\right) t\right] . \quad([V .1-41)
$$

The phases that appear explicitly here may augment the phase of a travelling wave mode of the field, to produce a polarization field at a frequency and in direction that differs from the electric field. This property will be discussed below. 


\section{IV.2 Polarization Modes}

Having obtained a formal prescription for evaluating the distribution of dipole moments that constitute the polarization field, we must next apportion this field into modes in order to determine the portions of the polarization field that affect separately each of the electric field modes.

\section{Positive Frequency Components}

The dipole moment, like the electric field and the polarization field, can be separated into positive and negative frequency part:

$$
\langle d(r, t)\rangle=\left\langle d^{(+)}(r, t)\right\rangle+\left\langle d^{(-)}(r, t)\right\rangle .
$$

The positive frequency part $e^{(+)}(r, t)>$ is, in the above summations, the portion that involves $\mathscr{E}_{\lambda}(\mathrm{r}, \mathrm{t}) \exp \left(i \mathrm{k}_{\lambda} \cdot \mathrm{r}-j \omega_{\lambda} t\right)$ as a factor. The positive-frequency portion of the dipole moment can be written (with the neglect of single-photon resonances)

$$
\begin{aligned}
& \left\langle d^{(+)}(r, t)=\sum_{p p^{\prime} \lambda^{\prime}} \rho_{p p^{\prime}}(r, t)<p\left|X\left(\omega_{\lambda}\right)\right| p\right\rangle . E^{(+)}{ }_{\lambda}(r, t) \\
& x \exp \left\{i\left(\mathbf{K}_{\mathbf{p}}-\mathbf{K}_{\mathbf{p}^{1}}\right), \mathbf{r}-i\left(\mathbf{W}_{\mathbf{p}}-\mathbf{W}_{\mathbf{p}^{\prime}}\right) t\right\}
\end{aligned}
$$

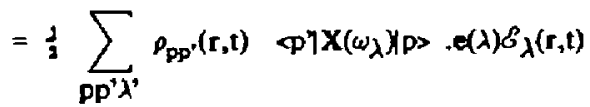

$$
\begin{aligned}
& x \exp \left[-i\left(W_{p}-W_{p^{\prime}}+w_{\lambda}\right) t\right] \exp \left[i\left(\mathbf{K}_{p}-\mathbf{K}_{p^{\prime}}+\mathbf{k}_{\lambda}\right) \mathbf{r}\right] .
\end{aligned}
$$

If the density matrix is a slowly varying function of time, in the Dirac picture, then this expression shows that the dipole moment has frequency components at the values

$$
\omega=W_{p}+\omega_{\lambda}-W_{p^{\prime}}
$$

The frequencies $0-\omega_{\lambda}$ are the frequencies of various Raman transitions. We can also recognize that, if the density matrix varies slowly with position, as it will when the effective Hamiltonian varies slowly, then the dipole moments at different positions are related by an exponentiated phase factor. In other words, the induced dipole momea? has properties of travelling waves. (This molecular excitation wave is sometimes termed the $Q$ wave.) By our choice of vectors $K_{p}$ as directed along the dominant radiation direction, the dipole waves also travel in this direction (when $\phi(r, t)$ is slowly varying in position and time).

\section{The Polarization-Field Modes}

We can use this expression for the positive frequency part of the dipole moment to evaluate the pulse-mode expansion of the polarization field. We start from the basic connection, at fixed position $\mathbf{r}$, 


$$
P(+)(r, t)=\mathcal{N}(r, t)<d^{(t)}(r, t) b .
$$

By means of the adiabatic approximation developed above we express the dipole moment in terms of the electric-field mode expansions. For a single molecular species we obtain the result

$$
\begin{aligned}
& P^{(t)}(r, t)=\mathcal{N}(r, t) \sum_{p p^{\prime} \lambda} \rho_{p p^{\prime}}(r, t)<p \eta X\left(\omega_{\lambda}\right)|p\rangle, E^{(+)} \lambda_{\lambda}(r, t) \\
& x \exp \left(K\left(\mathbf{K}_{p^{\prime}}-\mathbf{K}_{p^{\prime}}\right) \cdot r-i\left(W_{p}-W_{p^{\prime}}\right) t\right] \\
& =\mathcal{N}(r, t) \frac{1}{2} \sum_{\rho p^{\prime} \lambda} \rho_{p p^{\prime}}(r, t)\left\langle p^{\dagger}\right| X\left(\omega_{\lambda} X \rho\right\rangle . e(\lambda) \mathscr{E}_{\lambda}(r, t) \\
& x \exp \left(i\left(\mathbf{K}_{\mathbf{p}}-\mathbf{K}_{p}+k_{\lambda}\right) \cdot I-i\left(W_{p}-W_{p}+w_{\lambda}\right) t\right] .
\end{aligned}
$$

This has the appearance of 3 summation over a discrete set of frequencies appearing as arguments of the exponential. However, as written, it applies to a single nolecular species. To complete the macroscopic average we must include an additional summation over Doppler velocities of the molecules. When this is done, the frequencies are no longer discrete, but form Doppler-broadended lines.

The set of lines that occur in the frequency sum may be obained by the followins construction. Suppose we consider a sel of electric field frequencies, Starting from each of those frequencies, te form other frequencies by adding all possible combinations $W_{p}-W_{p}$. The result will be a list of discrete frequencies, those of the original set plus all possible increments of single molecular Bohr frequencies. (Thase transitions constitute the set of Stokes and anti-Stokes frequencies.) Then, using this enlarged list, we tepeat the construction of all possible combinations with single Bohr frequencies. We thereby obtain an enlarged list, comprising second, third, and higher order Stokes frequencies, and second, third, and higher order anti-Slokes frequencies. (In this reckoning the ordinary Stokes line is termed the first Stokes) By continuing this procecure we prepare a jengthy catalog of discrete frequencies. Associated with each frequency are two polarization vectors. All of these frequencies and polarization vectors may appear in the summation that here defines the polariation field $P(t)$.

Let us compare this expression, valid for any form of the pake expansion, with the requirement that the polarization field have the pulse-mode expansion

$$
p^{\prime+)}(r, t)=\frac{3}{2} \sum_{\lambda} e(\lambda) \mathscr{F}_{\lambda}(r, t) \exp \left(i k_{\lambda}-r-i \omega_{\lambda} t\right) .
$$

The carrier wavevectors and frequencies are assumed to be those used in the electric field expansion, Eqn. (V.1-4). As in that expansion, the connection between $k_{\lambda}$ and $\omega_{\lambda}$ remains to be defined. The result of the comparison is 


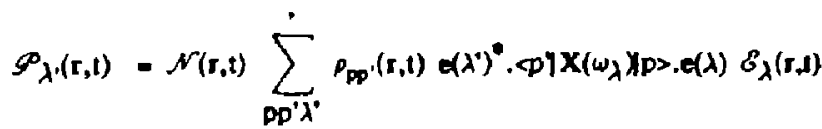

$$
\begin{aligned}
& x \exp \left[i\left(K_{p}-K_{p^{\prime}}+k_{\lambda}-k_{\lambda^{\prime}}\right), r\right] \exp \left[-i\left(W_{p^{\prime}}-W_{p^{\prime}}+\omega_{\lambda}-\omega_{\lambda}\right) t\right] .
\end{aligned}
$$

Before commenting on the significance of this expression, and particularly on the implicit restrictions upon $p p^{\prime} \lambda$ it is useful to introduce a tensor that incorporates the directionality properties of the vector fields.

\section{The E Field Polarization Tensor}

The expression for the polarization mode involves the dot product of the unit vector $e\left(\lambda^{\prime}\right)$ with the vector $X(\omega) . e(\lambda)$ obtained, in turn, from the dot product of the dyadic $X(\omega)$ with the vector $e(\lambda)$. Expressed by means of Cartesian coordinates upon the tensor and vectors, the required scalar is

$$
e\left(\lambda^{\prime}\right)^{\star} \cdot X(\omega) \cdot e(\lambda)=\sum_{i j} x_{i j}(\omega) e_{i}\left(\lambda^{\prime}\right)^{*} e_{j}(\lambda) .
$$

Here, for example, $e_{x}(\lambda)$ is the $x$ component of the unit vector $(\lambda)$. The directional relationship between the polarization field in mode $\lambda^{*}$ and the eloctric field in mode $\lambda$ accurs through the bilinear combinations of unit-vector amplitudes $e_{i}\left(\lambda^{\prime}\right)^{*} e_{j}(\lambda)$. In writing this sum it is useful to introduce the polarization dyadic $E\left(\lambda, \lambda^{\prime}\right)$ constructed from mode unit vectors

$$
E\left(\lambda^{*}, \lambda\right)=e\left(\lambda^{*}\right)^{*} \theta(\lambda) \quad \text { or } \quad E_{i j}\left(\lambda^{\prime}, \lambda\right)=e_{i}\left(\lambda^{\prime}\right)^{*} e_{j}(\lambda) \text {. }
$$

Just as $X(w)$ is a dyadic constructed from a pair of atomic dipole-moment vectors, so too is $E\left(\lambda^{\prime}, \lambda\right)$ a dyadic constructed from two unit vectors.

\section{The Polarization Mode Envelope}

By introducing the polarization tensor we can write the formula for the polarization-field mode envelope, for a single molecular species, as

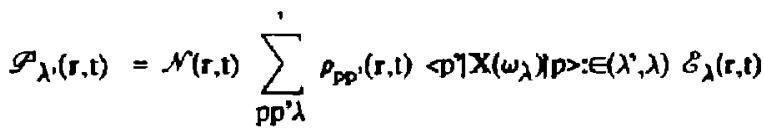

$$
\begin{aligned}
& x \exp \left[i\left(\mathbf{K}_{\mathbf{p}}-\mathbf{K}_{\mathbf{p}^{\prime}}+\mathbf{k}_{\lambda^{\prime}}-\mathbf{k}_{\lambda^{\prime}}\right) \cdot \mathbf{r}\right] \exp \left[-i\left(\mathbf{W}_{\mathbf{p}}-W_{p^{\prime}}+\omega_{\lambda^{\prime}}-\omega_{\lambda^{\prime}}\right) t\right\}
\end{aligned}
$$

This is a matrix equation in the mode indices $\lambda^{\prime}, \lambda$, in the molecular indices $\mathbf{p}, \mathbf{p}^{\prime}$, and, implicitly, in some (arbitrary) spatial reference system for the unit vectors, say a Cartesian system.

The sum that occurs here is over some relatively modest number of discrete frequencies appropriate to the electric field pulses. Associated with each carrier fre- 
quency $w_{\lambda}$ is a bandzidth $\&_{\lambda}$. We want to essociate with the frequency $w_{\lambda}$, all of those frequencies in Eqn. (IV 2-11) that lie within the bandwidth $\delta w_{\lambda \text {. }}$ As a consequence of this restriction the summation over molecular states $p$ and $p^{\prime}$ and over mode frequencies is restricted by the constraint

$$
\left|w_{\lambda^{*}}+w_{p^{\prime}}-w_{p}-w_{\lambda}\right|<w_{\lambda-}
$$

This condition may be regarded as a consistancy requirement. Were we to discover that our ratalog of mode frequencies produced, through the interaction of the pulses with molecules, a set of elosely spaced discrete frequencies then we might need w redefine the relevant mode; the redefined envelope will represent modulation of a carrier by closely spaced sidebands. If we are to apply the notion of pulse modes in wavevectors as well as in frequencies, then similar reasoning must apply to the spatial exponential. We must have a sum over well-separated directions in order to define distinct mode beams. This means that the sum is further restricted by the requirement

$$
\left|\mathbf{k}_{\lambda^{\prime}}+\mathbf{k}_{\mathbf{p}^{\prime}}-\mathbf{k}_{\lambda}-\mathbf{k}_{\mathbf{p}}\right|<\delta \mathbf{k}_{\lambda^{\prime}}
$$

where $\delta k_{\lambda}$, is the spread in wavevectors in mode $\lambda^{*}$.

The Susceptibility Matrix

One of ten expresses the general relationship between the polarization field $P$ and the electric field $E$ by means of a susceptibility tensor $x$, through the definition

$$
\mathbf{P}=x \mathbf{E} \text {. }
$$

For monochromatic radiation this expression takes the form

$$
\mathbf{P}^{(+)} \omega=x(\omega) \mathbf{E}^{(+)} \omega \cdot
$$

We can generalize this definition to treat pulse modes by writing

$$
\mathscr{P}_{\lambda}(r, t)=\sum_{\lambda^{\prime}} x_{\lambda \lambda^{\prime}}(r, t) \mathscr{\delta}_{\lambda^{\prime}}(r, t)
$$

The susceptibility matrix needed bere is obtainable from our expression above. For a single molecular species it is

$$
\begin{aligned}
& x_{\lambda \lambda},(r, t)=\mathcal{N}(r, t) \sum_{p p^{*}} p_{p_{p}}(r, t)<p \mid X\left(\omega_{\lambda}, y^{\prime} p^{\prime}>: \in\left(\lambda, \lambda^{\prime}\right)\right. \\
& x \exp \left[i\left(K_{p^{\prime}}-K_{p}+k_{\lambda^{\prime}}-k_{\lambda}\right) \cdot r\right] \exp \left[-i\left(W_{p^{\prime}}-W_{p}+\omega_{\lambda} \lambda^{\prime}-\omega_{\lambda}\right) t\right] .
\end{aligned}
$$

The summation restrictions are as discussed above. For multiple species (e.g. Doppler distributions or different chemical species) the formula requires appropriate summation or integration over distributions. 
This formula is based on adiabatic elimination of nonresonant states (the $Q$ states). It is based on the assumption that pulses have negligible frequency components that satisfy resonance conditions between the $P$ states of interest and the presumably unpopulated $Q$ states. Corrections for finite bandwith provide formulas that involve time derivatives of the pulse envelope. The simplest of these produces the rerised formula

$$
\mathscr{P}_{\lambda}(r, t)=\sum_{\lambda^{\prime}} x_{\lambda \lambda^{\prime}}(r, t) \mathscr{E}_{\lambda^{\prime}}(r, t)+j \sum_{\lambda^{\prime}} x^{(1)} \mu^{\prime}(r, t) \frac{\partial}{\partial t} E_{\lambda^{\prime}}(r, t)
$$

where

$$
\begin{gathered}
x^{(1)} \lambda^{\prime}(r, t)=\mathcal{N}(r, t) \sum_{p p^{\prime}} f_{p^{\prime} p^{\prime}}(r, t)\left[\frac{\partial}{\partial \omega}\left\langle p|X(\omega)| p^{\prime}\right\rangle\right]_{\omega=\omega_{\lambda}}: E\left(\lambda, \lambda^{\prime}\right) \\
\quad x \exp \left\{i\left(K_{p^{\prime}}-K_{p}+k_{\lambda^{\prime}}-k_{\lambda}\right) \cdot r\right] \exp \left[-i\left(W_{p^{\prime}}-W_{p^{\prime}}+\omega_{\lambda^{\prime}}-\omega_{\lambda}\right) r\right] .
\end{gathered}
$$

The second sum, involving the time derivarive, may be expected to be smaller than the first term by roughly the ratio of pulse bandwidth $\Delta \omega_{\lambda}$ to the detuning $\left|W_{g}-W_{p}-\omega_{\lambda}\right|$ between the carrier frequency and a representative pair of levels from $P$ and $Q$ space. When this ratio is not negligibly small, dynamics of some $Q$ states may become significant, and probability does not remain confined entirely to the $P$ space. 
The polarization-field pulse envelope $\mathscr{P}_{\lambda}$ appears as the source terni in an equation for the mode-envelope $\mathscr{E}_{\lambda}$ of the electric field. $A$ variety of equations can be written for this field. The simplest approach is to regard the polarization field as expressing all possible molectular response to the imposed radiatios, pitsuivi atienijising a priori to separate the polarization field into a linear and a nonlinear portion. With this approach we do not introduce, a priori, an index of refraction. The mode equation for expansions with constant $k_{\lambda}$ and $\omega_{\lambda}$ (independent of space and time) reads

$$
\begin{gathered}
{\left[\hat{\mathbf{k}}_{\lambda} \cdot \nabla+\frac{\omega_{\lambda}}{c^{2} k_{\lambda}} \frac{\partial}{\partial t}-\frac{\left.\pi\left(\omega_{\lambda}\right)^{2}-\left(c k_{\lambda}\right)^{2}\right]}{2 c^{2} k_{\lambda}}-\frac{i}{2 k_{\lambda}}\left(\nabla^{2}-\frac{1}{c^{2}} \frac{\partial^{2}}{\partial t^{2}}\right)\right] \mathscr{G}_{\lambda}(r, t)} \\
=\frac{2 \pi i\left(\omega_{\lambda}\right)^{2}}{c^{2} k_{\lambda}}\left[1+\frac{2 i}{\omega_{\lambda}} \frac{\partial}{\partial t}-\frac{1}{\left(\omega_{\lambda}\right)^{2}} \frac{\partial^{2}}{\partial t^{2}}\right] \mathscr{P}_{\lambda}(r, t)
\end{gathered}
$$

where $\hat{k}_{\lambda}$ is a unit vector along $k_{\lambda}$. In deriving this equation we have not yet assuned a slowly varying envelope (or eliminated backward waves) nor have we introduced any particular connection between $k_{\lambda}$ and $w_{\lambda}$.

\section{The Electric Field Equations}

It is evident from the preceding paragraphs that for Raman processes the polarization envelope $\mathscr{F}_{\lambda}$ depends explicitly on each of the field modes $\mathscr{E}_{\lambda^{\prime}}$. There is also an implicit dependence on the electric fields through the dependence of the density matrix upon these fields. By exhibiting this relationship by means of a susceptibility matrix $x_{x \lambda}$, as in Eqn. (IV.2-15), we obtain an equation involving only the various electric field envelopes,

$$
\begin{aligned}
& {\left[\hat{\mathbf{k}}_{\lambda} \cdot \nabla+\frac{\omega_{\lambda}}{c^{2} k_{\lambda}} \frac{\partial}{\partial t}-\frac{\left.\pi\left(\omega_{\lambda}\right)^{2}-\left(c k_{\lambda}\right)^{2}\right]}{2 c^{2} k_{\lambda}}-\frac{i}{2 k_{\lambda}}\left(\nabla^{2}-\frac{1}{c^{2}} \frac{\partial^{2}}{\partial \tau^{2}}\right)\right] \delta_{\lambda}(r, t)} \\
& =\sum_{\lambda^{\prime}}\left[\frac{2 \pi i\left(\omega_{\lambda}\right)^{2}}{c^{3} k_{\lambda}}-\frac{4 \pi\left(\omega_{\lambda}\right)}{c^{2} k_{\lambda}} \frac{\partial}{\partial t}-\frac{2 \pi i}{c^{2} k_{\lambda}} \frac{\partial^{2}}{\partial t^{2}}\right] x \lambda \cdot(\tau, 1) \mathscr{E}_{\lambda} \cdot(\tau, t) .
\end{aligned}
$$

Upon evaluating the time derivatives we can write this equation as 


$$
\begin{aligned}
& {\left[\hat{\mathbf{k}}_{\lambda} \cdot \nabla+\frac{\omega_{\lambda}}{c^{2} k_{\lambda}} \frac{\partial}{\partial t}-\frac{\left.\pi\left(\omega_{\lambda}\right)^{2}-\left(c k_{\lambda}\right)^{2}\right]}{2 c^{2} k_{\lambda}}\right] g_{\lambda}(r, t)-\frac{i}{2 k_{\lambda}}\left(\nabla^{2}-\frac{1}{c^{2}} \frac{\partial^{2}}{\partial^{2}}\right) E_{\lambda}(r, t)}
\end{aligned}
$$

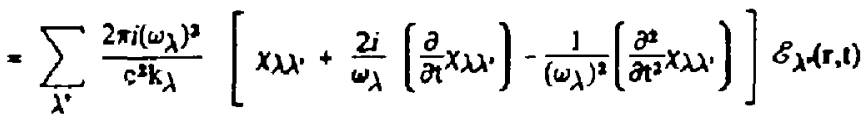

$$
\begin{aligned}
& -\sum_{\lambda^{\prime}} \frac{4 \pi \omega_{\lambda}}{c^{2} k_{\lambda}}\left[x \mu^{\prime}+\frac{i}{\omega_{\lambda}}\left[\frac{\partial}{\partial t^{\prime}} x_{\lambda \lambda^{\prime}}\right)\right] \frac{\partial}{\partial t} E_{\lambda^{\prime}}(r, t) \\
& -\sum_{\lambda^{\prime}} \frac{2 \pi i}{c^{2} \mathbf{k}_{\lambda}} x_{\lambda \lambda} \cdot \frac{\partial^{3}}{\partial t^{2}} \mathscr{g}_{\lambda,}(\mathbf{r}, \mathrm{t})
\end{aligned}
$$

\section{The Gain Matrix}

The sum on the right hand side includes the case $\lambda=\lambda$. Let us extract this term and place it on the left hand side. Let us introduce a complex-valued gain matrix $G$ whose elements $G_{\lambda}$, are indexed by the mode labels, and a complex-valued mode velocity matrix $1 / v_{\lambda \lambda^{\prime}}(r, t)$ through the definitions

$$
\begin{aligned}
& G_{\lambda \lambda^{\prime}(r, t)}=\frac{2 \pi i\left(\omega_{\lambda}\right)^{2}}{c^{2} k_{\lambda}}\left[1+\frac{2 i}{\omega_{\lambda}} \frac{\partial}{\partial t}-\frac{1}{\left(\omega_{\lambda}\right)^{2}} \frac{\partial^{2}}{\partial t^{2}}\right] x_{\lambda \lambda^{\prime}}(r, t) . \\
& \frac{1}{v_{\lambda \lambda^{\prime}}(r, t)}=\frac{\omega_{\lambda}}{c^{2} k_{\lambda}}\left[\delta\left(\lambda, \lambda^{\prime}\right)+\left(1+\frac{i}{\omega_{\lambda}} \frac{\partial}{\partial t}\right) 4 \pi \chi \lambda \mu \cdot(r, t)\right]
\end{aligned}
$$

With these definitions we can write the mode equation as

$$
\begin{gathered}
{\left[\hat{\mathbf{k}}_{\lambda} \cdot \nabla+\frac{1}{v_{\lambda \lambda}(\mathbf{r}, t)} \frac{\partial}{\partial t}-G_{\lambda \lambda}(r, t)-\frac{\left.\pi\left(\omega_{\lambda}\right)^{2}-\left(c_{\lambda}\right)^{2}\right]}{2 c^{2} k_{\lambda}}-\frac{i}{2 k_{\lambda}}\left[\nabla^{1}-\frac{1}{c^{2}}\left(1+4 \pi \lambda_{\lambda \lambda}\right) \frac{\partial^{2}}{\partial t^{2}}\right)\right] E_{\lambda}(r, t)} \\
=\sum_{\lambda^{\prime} \neq \lambda}\left[G_{\lambda \lambda \cdot}(r, t)-\frac{1}{v_{\lambda \lambda^{\prime}}(r, t)} \frac{\partial}{\partial t}-\frac{2 \pi i}{c^{2} k_{\lambda}} x \lambda \lambda^{\prime}(r, t) \frac{\partial^{2}}{\partial t^{2}}\right] E_{\lambda}(r, t) .
\end{gathered}
$$

We can use the expression (IV.2-16) for the susceptibility matrix to evaluate the definitions of $G_{\lambda \lambda^{\prime}}$ and $v_{\lambda j}$, for a single molecular species (a more complete definition will include integration over a Doppler distribution of moving molecules). For example, with the neglect of second derivatives we obtain the expressions 


$$
\begin{aligned}
& {\left[\hat{k}_{\lambda} \cdot \nabla+\frac{1}{v_{\lambda \lambda}(r, t)} \frac{\partial}{\partial}-G_{\lambda \lambda}(r, t)-\frac{\left.\pi\left(\omega_{\lambda}\right)^{2}-\left(c k_{\lambda}\right)^{2}\right]}{2 c^{2} k_{\lambda}}\right] g_{\lambda}(r, t)} \\
& =\sum_{\lambda^{\prime} \neq \lambda}\left[G_{\mu \cdot(r, t)}-\frac{1}{v_{\lambda \cdot(r, t)}} \frac{\partial}{\partial t}\right] E_{\lambda^{\cdot} \cdot(r, t)} \\
& G_{\lambda \lambda^{\prime}}(\mathrm{r}, \mathrm{t})=\frac{2 \pi i\left(\omega_{\lambda}\right)^{2}}{\mathrm{c}^{2} \mathrm{k}_{\lambda}} \mathscr{N}(\mathrm{r}, \mathrm{t}) \sum_{\mathrm{pp}^{\prime \prime}}\left\langle\mathrm{P} \mathrm{X}\left(\omega_{\lambda}\right) \lambda \rho^{\prime}>: E\left(\lambda, \lambda^{\prime}\right)\right. \\
& x \exp \left[i\left(\mathbf{K}_{\mathrm{p}^{\prime}}-\mathbf{K}_{\mathrm{p}}+\mathbf{k}_{\lambda^{\prime}}-\mathbf{k}_{\lambda}\right) \cdot \mathbf{r}\right] \exp \left(-i\left(\mathbf{W}_{p^{\prime}}-W_{\mathrm{p}^{\prime}}+\omega_{\lambda^{\prime}}-\omega_{\lambda}\right) r\right] \\
& x\left[1+\frac{2\left(w_{p^{\prime}}-w_{p}-w_{\lambda}+w_{\lambda^{\prime}}\right)}{w_{\lambda}}+\frac{2 i}{w_{\lambda}} \frac{\partial}{\partial t}\right] \rho_{p^{\prime} p}(r, t)
\end{aligned}
$$

The term involving the ratio of frequency differences to mode frequency is much less than unity and can be neglected. Tr evaluate the time derivative we can use the equation of motion for the density matrix. Let $h W$ be the effective Hamiltonian (that is, the Raman Hamilionian) and $\mathrm{r}$ be the relaxation matrix, as used in the equation of motion for the Dirac-picture density matrix [cf. the section excit\}

$$
\frac{\partial}{\partial t} \rho=-i(W p-\rho W)-\Gamma \rho
$$

(Explicit expressions for $W$ are not needed here; they are described in a separate section, excit.) Then we can rewrite the gain matrix as

$$
\begin{aligned}
& G_{\lambda \lambda,}(r, t)=\frac{2 \pi i\left(\omega_{\lambda}\right)^{2}}{c^{2} k_{\lambda}} \quad r(r, t) \sum_{p p^{\prime}}^{\prime}\langle p| X\left(\omega_{\lambda}, X p^{\prime}>: E\left(\lambda, \lambda^{\prime}\right)\right. \\
& x \exp \left[i\left(K_{p^{\prime}}-K_{p^{\prime}}+k_{\lambda^{\prime}}-k_{\lambda}\right) \cdot r\right] \exp \left[-r\left(W_{p^{\prime}}-W_{p^{\prime}}+\omega_{\lambda^{\prime}}-\omega_{\lambda}\right) t\right] \\
& x\left[\left(1-\frac{2 T_{E^{\prime} P}}{\omega_{\lambda}}\right) p_{p_{p}+}+\frac{2}{w_{\lambda}}\left[W_{\rho p}\right]_{p p}\right]
\end{aligned}
$$

For conventional fields (i.e. fields for which interaction energies d.E are much less than photon energies tiu) we can neglect the ratios of interaction energies to photon energies, $W_{2 b} / \omega_{\lambda}$, and 50 neglect the commutator $[W, \rho]$. The rekatation term, involving $\Gamma$, leads to attenuation of the radiation. It represents energy lost from the molecule into a thermal bath.

The real part of a diagonal element of the gain matrix is responsible for absorption or gain of a pulse in the absence of other modes. The imaginary part produces a phase shift. The two parts together are 


$$
\begin{aligned}
& G_{\mu(r, t)}=\frac{2 x\left(\omega_{\lambda}\right)^{2}}{\epsilon^{3} k_{\lambda}} \mathscr{N}(r, t) \sum_{p p^{\prime}} \delta\left(w_{p}, W_{p^{\prime}}\right)<p \mid X\left(\omega_{\lambda}, p^{\prime}>: E(\lambda, \lambda)\right. \\
& x \exp \left[i\left(K_{p^{\prime}}-K_{p}\right) \cdot r\right]\left[\left(i+\frac{2 \Gamma_{p^{\prime} p}}{\omega_{\lambda}}\right) \rho_{p^{\prime} p^{\prime}}+\frac{2 i}{w_{\lambda}}\left[W_{, \rho}\right]_{p_{p}}\right]
\end{aligned}
$$

The summation goes over degenerate states. The complex-valued mode velocity has a similar expression,

$$
\begin{aligned}
& \frac{1}{v_{\mu \lambda}(r, t)}=\frac{\omega_{\lambda}}{c^{2} k_{\lambda}}+\frac{4 \pi \omega_{\lambda}}{c^{2} k_{\lambda}} \mathscr{r}(r, t) \sum_{p p^{\prime}} \delta\left(W_{p}, W_{p^{\prime}}\right)\left\langle p\left|X\left(\omega_{\lambda}\right)\right| p^{\prime}>: E(\lambda, \lambda)\right. \\
& x \exp \left[i\left(\mathbf{K}_{p^{\prime}}-K_{p}\right) \cdot r\right]\left[\left(1-\frac{\left(T_{p^{\prime} P}\right)}{\omega_{\lambda}}\right) \rho_{p_{p}}+\frac{1}{\omega_{\lambda}}\left[W_{, \rho}\right]_{p^{\prime} p}\right]
\end{aligned}
$$

[Note that the off-diagonal elements of $1 / v$ are smailer than the diagonal elements by the ratio $4 \pi x /(1+4 \pi x)$ ]. Each of these expressions refers to a single species. To treat Doppler broadening or other varieties of molecules one must generalize these expressions to sum and integrate over relevant distributions.

\section{The Refractive Index}

We have not yet specified the connection between $k_{\lambda}$ and $\omega_{\lambda}$, as expressed by the relationship $c k_{\lambda}=\eta_{\lambda} \omega_{\lambda}$ where $\eta_{\lambda}$ is the refractive index for mode $\lambda$. The choice is, in a sense, arbitrary, because the results of integration (if done exactly) will compensate for our choice. We wish to choose $\eta_{\lambda}$ to simplify the propagation equations as much as possible. The most suitable choice for $i \lambda$ is given by

$$
\begin{aligned}
& \eta_{\lambda}(r, i)^{2}=1-\frac{2 i\left(c^{2} k_{\lambda}\right)}{\left(\omega_{\lambda}\right)^{2}} G_{\lambda \lambda}(r, t)=1+\left[1+\frac{2 j}{\omega_{\lambda}} \frac{\partial}{\partial t}\right] 4 \pi \times \lambda \lambda(r, t) \\
& =1+4 \pi \mathcal{N}(r, t) \sum_{p p^{\prime}} \delta\left(W_{p}, W_{p^{\prime}}\right)<p \mid X\left(\omega_{\lambda}\right)^{\prime} p^{\prime}>: E(\lambda, \lambda) \\
& x \exp \left[i\left(\mathbf{K}_{\mathbf{p}^{\prime}}-\mathbf{K}_{\mathbf{p}}\right) \cdot \mathbf{r}\right]\left[\left(1-\frac{i 2 \Gamma_{p_{p}^{p}}}{\omega_{\lambda}}\right] \rho_{p_{p}^{\prime}}+\frac{2 i}{\omega_{\lambda}}\left[W_{p} \rho l_{p p}\right]\right.
\end{aligned}
$$

This choice eliminates the constant coefficient of $E_{\lambda}$. With the neglect of second derivatives this choice provides the propagation equation 


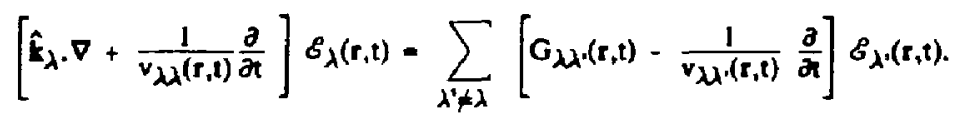

This is the desired equation for propagation of shon pulses through a Raman-active medium. The equation introduces a mode velocity and, implicitly, a ref ractive index, for each mode. These depend on space and time only slowly (over scales large compared with $k_{\lambda}$ and $\left.\omega_{\lambda}\right)$, through the variation of the density matrix. The completed description of propagation must include some procedure for determining the density matrix, either by solving transient excitation equations or by considering steady state values.

\section{The Mode Velocity}

We may note that if we neglect spatial variations of the material medium and time variation of the density matrix (presumed to be slowly varying at most), we obtain a mode velocity that is independent of space and time. Under those conditions the equation for a single mode, in the absence of other modes, is

$$
\left[\hat{\mathbf{k}}_{\lambda} \cdot \nabla+\frac{1}{v_{\lambda \lambda}} \frac{\partial}{\partial t}\right] \mathscr{G}_{\lambda}(\tau, t)=0
$$

This is a nondispersive equation for the pulse: it does not describe pulse reshaping. To produce dispersive propagation the pulse must modify the populations. It is the differing nolecular excitation for different frequencies that produces dispersive effects.

Using the derinition of $\eta_{\lambda}$ we can write the mode velocity as

$$
\begin{aligned}
\frac{1}{v_{\lambda \lambda}(r, t)} & =\frac{\eta_{\lambda}}{c}\left[1-\frac{i}{\omega_{\lambda} \eta_{\lambda}} \frac{\partial}{\partial t} 4 x_{\lambda \lambda}(r, t)\right] \\
& =\frac{\eta_{\lambda}}{c}\left[1-\frac{2 i}{\omega_{\lambda} \eta_{\lambda}} \frac{\partial}{\partial t} \ln \eta_{\lambda}\right]
\end{aligned}
$$

We see that, apart from time variation of the susceptibility (originating in population changes), the mode velocity is the phase velocity. When the populations change slowly over an optical cycle (this is the situation we consider) the velocity expression becomes

$$
\frac{1}{v_{\lambda \lambda}(r, t)} \simeq \frac{\eta_{\lambda}}{c}=\frac{k_{\lambda}}{w_{\lambda}}
$$

This velocity depends upon position and time as a consequence of density variations and population changes. 


\section{\$IY.4 Summary}

We have shown that when a mode expansion is made for the eloctric field, in the form of envelopes modifying fixed carrier waves,

$$
E^{(+)}(r, t)=\frac{1}{2} \sum_{\lambda} c(\lambda) E_{\lambda}(r, t) \exp \left[i k_{\lambda}, r-i \omega_{\lambda} t\right]
$$

the induced dipole moment can be expressed as a superposition of modulated carrier waves, in the form

$$
\begin{aligned}
& \left\langle\mathrm{d}^{(+)}(r, 1)\right\rangle=\frac{1}{2} \sum_{\mathrm{pp} p^{\prime} \lambda} \rho_{\mathrm{pp}}(r, t)\langle\mathrm{p}\rceil X\left(\omega_{\lambda}\right)|p\rangle . e(\lambda) \mathscr{E}_{\lambda}(r, t) \\
& x \exp \left[i\left(\mathbf{K}_{\mathbf{p}}-\mathbf{K}_{\mathbf{p}^{\prime}}+\mathbf{K}_{\lambda}\right) \cdot \mathbf{r}\right] \exp \left[-i\left(W_{\mathbf{p}}-\mathbf{W}_{\left.\left.\mathbf{p}^{\prime}+w_{\lambda}\right) t\right]}\right.\right.
\end{aligned}
$$

Consequently the field envelope functions, in the absence of single-photon resonances, obey the mode equations

$$
\left[\hat{\mathbf{k}}_{\lambda} \cdot \nabla+\frac{1}{v_{\lambda \lambda^{\prime}}(r, t)} \frac{\partial}{\partial t}\right] \mathscr{E}_{\lambda}(r, t)=\sum_{\lambda^{\prime} \neq \lambda}\left[G_{\lambda^{\prime}}(r, t)-\frac{1}{v_{\lambda \lambda^{\prime}}(r, t)} \frac{\partial}{\partial t}\right] E_{\lambda^{\prime}}(r, t)
$$

The gain matrix $G_{\lambda \mu}$ and the mode velocity $v_{\lambda \lambda}$, that appear in this equation are each complex-valued functions of position and time, given (with the neglect of $\left[W_{\rho} \rho / \omega_{\lambda}\right.$ ] by the expressions

$$
\begin{aligned}
& G_{\lambda \lambda^{\prime}}(r, t)=\frac{2 \pi i \omega_{\lambda}}{c \eta_{\lambda}} \mathscr{N}(r, t) \sum_{p p^{\prime}}^{\prime} \rho_{p^{\prime} p}(r, t)<p \mid X\left(\omega_{\lambda}\right), p^{\prime}>: E\left(\lambda, \lambda^{\prime}\right)\left[1-\frac{2 r \Gamma_{p^{\prime} P}}{\omega_{\lambda}}\right] \\
& \pi \exp \left[i\left(\mathbf{K}_{\mathbf{p}^{\prime}}-\mathbf{K}_{\mathbf{p}}+\mathbf{k}_{\lambda^{\prime}}-\mathbf{k}_{\lambda}\right) \cdot r\right] \exp \left[-i\left(\mathbf{W}_{\mathbf{p}^{\prime}}-\mathbf{W}_{\mathbf{p}}+\boldsymbol{\omega}_{\lambda^{\prime}}-\omega_{\lambda}\right) t\right] \\
& \frac{1}{v_{\lambda \lambda^{\prime}}(r, t)}=\frac{\delta\left(\lambda, \lambda^{\prime}\right)}{c \eta_{\lambda}}+\frac{4 \pi \mathcal{N}(r, t)}{c \eta_{\lambda}} \sum_{p p^{\prime}} \rho_{p^{\prime} p}(r, t)<p^{\prime} \mid X\left(\omega_{\lambda^{\prime}} y^{\prime} p^{\prime}>: \in\left(\lambda_{\lambda} \lambda^{\prime}\right)\left[1-\frac{i\left(\Gamma_{p^{\prime} p}\right)}{\omega_{\lambda}}\right]\right. \\
& x \exp \left[i\left(\mathbf{K}_{\mathbf{p}^{\prime}}-\mathbf{K}_{\mathbf{p}}+\mathbf{k}_{\lambda^{\prime}}-\mathbf{k}_{\lambda}\right) \cdot \mathbf{r}\right] \exp \left[-i\left(W_{p^{\prime}}-W_{p^{\prime}}+\omega_{\lambda^{\prime}}-\omega_{\lambda}\right) t\right]
\end{aligned}
$$

(Note that the off-diagonal elements of $1 / v$ are smaller than the diagonal elements by the ratio $4 \pi x /(1+4 \pi x)]$. The relationship between $k_{\lambda}$ and $\omega_{\lambda}$ is taken to be a slowly varying function of space and time, 
BWS 23 Jul 87

Dolar-24

$$
c k_{\lambda}=\omega_{\lambda} \eta_{\lambda}(r, t)
$$

where the refractive index is

$$
\begin{aligned}
\eta_{\lambda}(r, t)=1+2 \pi \mathcal{N}(r, t) & \sum_{p p^{\prime}} \delta\left(W_{p}-W_{p^{\prime}}\right) \rho_{p p^{\prime}}(r, t)<p\left|X\left(\omega_{\lambda}\right)\right| p^{\prime}>: E(\lambda, \lambda) \\
& x\left[1-\frac{12 \Gamma_{p p}}{\omega_{\lambda}}\right] \exp \left[i\left(K_{p^{\prime}}-K_{p}\right) \cdot r\right]
\end{aligned}
$$

Note that the mode velocity and the square of the refractive index may be written

$$
\begin{aligned}
& \frac{1}{v_{\lambda \lambda}(r, t)}=\frac{\eta_{\lambda}}{c}\left[1-\frac{i}{\eta_{\lambda} \omega_{\lambda}} \frac{\partial}{\partial t} 4 \pi x_{\lambda \lambda}(r, t)\right] \\
& \eta_{\lambda}(r, t)^{2}=1+\left[1+\frac{2 i}{\omega_{\lambda}} \frac{\partial}{\partial t}\right] 4 \pi \times \lambda(r, t)
\end{aligned}
$$

where the diagonal elements of the susceptibility matrix are

$$
\begin{aligned}
& x, u^{(r, t)}=\mathscr{N}(r, t) \sum_{D p^{\prime}} \sigma\left(W_{p^{\prime}}-W_{p^{\prime}}\right) \rho_{p^{\prime} p}(r, t) \leftarrow \mathcal{T} X\left(\omega_{\lambda}\right) \lambda p^{\prime}>: E(\lambda, \lambda) \\
& x\left[1-\frac{i 2 \Gamma_{p_{p}}}{\omega_{\lambda}}\right] \exp \left[i\left(\mathbf{K}_{p^{\prime}}-\mathbf{K}_{p}\right) \cdot \mathbf{r}\right]
\end{aligned}
$$

Molecular properties enter these expressions as time- and space-varying elements of the density matrix $\rho_{p p}$ (both diagonal and off-diagonal elements may occur, but only amoogst degenerate states) and in fixed values of matrix elements of the polarizability operator $\mathbf{X}\left(\omega_{\lambda}\right)$. The formulas rely on adiabatic elimination to produce a Raman Hamiltonian and a polarizibility operator, but they do not assume that molecular response to the radiation is either weak or steady. The complete description of radiation propagation must combine these equations for field modes with equations for molecular excitation, either dynamic (i.e. the Schrödinger equation) or steady-state. 